\title{
A re-examination of the time constant of the oculomotor neural integrator in human
}

Khojasteh, Elham ; Bockisch, Christopher J ; Straumann, Dominik ; Hegemann, Stefan C A

\begin{abstract}
We studied the horizontal oculomotor neural integrator in healthy human subjects during gaze holding in darkness. We found large variability among subjects with respect to the estimated time constants and the integrator's null position. We also found that individual subjects could demonstrate significantly nonlinear drift velocities as a function of eye position. Nevertheless, a consistent trend did not emerge. Consequently, cross subject averaging eliminates idiosyncratic nonlinear patterns and the average can be approximated by a linear function inside the range that was tested.
\end{abstract}

DOI: https://doi.org/10.1109/EMBC.2012.6347036

Posted at the Zurich Open Repository and Archive, University of Zurich

ZORA URL: https://doi.org/10.5167/uzh-74703

Conference or Workshop Item

Published Version

Originally published at:

Khojasteh, Elham; Bockisch, Christopher J; Straumann, Dominik; Hegemann, Stefan C A (2012). A re-examination of the time constant of the oculomotor neural integrator in human. In: 34th Annual Internat. Conference of the IEEE Engineering in Medicin and Biology Society, San Diego, USA, August 2012. Insti, 4780-3.

DOI: https://doi.org/10.1109/EMBC.2012.6347036 


\title{
A Re-Examination of the Time Constant of the Oculomotor Neural Integrator in Human*
}

\author{
Elham Khojasteh, Christopher J. Bockisch, Dominik Straumann, and Stefan C. A. Hegemann
}

\begin{abstract}
We studied the horizontal oculomotor neural integrator in healthy human subjects during gaze holding in darkness. We found large variability among subjects with respect to the estimated time constants and the integrator's null position. We also found that individual subjects could demonstrate significantly nonlinear drift velocities as a function of eye position. Nevertheless, a consistent trend did not emerge. Consequently, cross subject averaging eliminates idiosyncratic nonlinear patterns and the average can be approximated by a linear function inside the range that was tested.
\end{abstract}

\section{INTRODUCTION}

The neural integrator (NI) of the oculomotor system refers to a distributed network of neurons in the brainstem and cerebellum that performs the mathematical temporal integration of eye movement related velocity commands. The existence of the NI was first conceived by Skavenski and Robinson [1] to account for the fact that the ocular motor nuclei carry both position and velocity information during head velocity driven vestibulo-ocular reflex movements. They suggested that head velocity information from the vestibular sensors is integrated in the brainstem to provide position commands. It is commonly accepted that horizontal integration is performed by a distributed network of neurons in the medial vestibular nuclei, the nucleus prepositus hypoglossi, and the cerebellum. The function of the NI is necessary to keep the eyes at a desired location following a saccade. Saccadic burst (or velocity pulse) should also be integrated to produce a step position command sent to the motor neurons. Otherwise the visco-elastic properties of the eye plant (the extraocular muscles, eye ball and the tissue, collectively) will rapidly move the eye back towards the null position with a time constant of $\sim 200 \mathrm{~ms}$. The integrator function is however not ideal, as in the absence of visual feedback the eye will drift back to the null position, albeit with a much larger time constant than that of the plant.

*Research supported by Swiss National Science Foundation. E. Khojasteh also receives the FQRNT scholarship from Quebec, Canada.

E. Khojasteh is with the Department of Otorhinolaryngology, Head and Neck Surgery, Vestibulo-Oculomotor Laboratory, University Hospital Zürich, CH-8091 Zürich, Switzerland (phone: +41-44-255-5594, email: elham.khojasteh@mail.mcgill.ca).

C.J. Bockisch is with the Departments of Neurology, Otorhinolaryngology, and Ophthalmology, University Hospital Zürich, CH8091 Zürich, Switzerland (email: chris.bockisch@usz.ch).

D. Straumann is with the Department of Neurology, University Hospital Zürich, CH-8091 Zürich, Switzerland (email: dominik.straumann@usz.ch).

S.C. Hegemann is with the department of Otorhinolaryngology, Head and Neck Surgery, University Hospital Zürich, CH-8091 Zürich, Switzerland (email: stefan.hegemann@usz.ch).

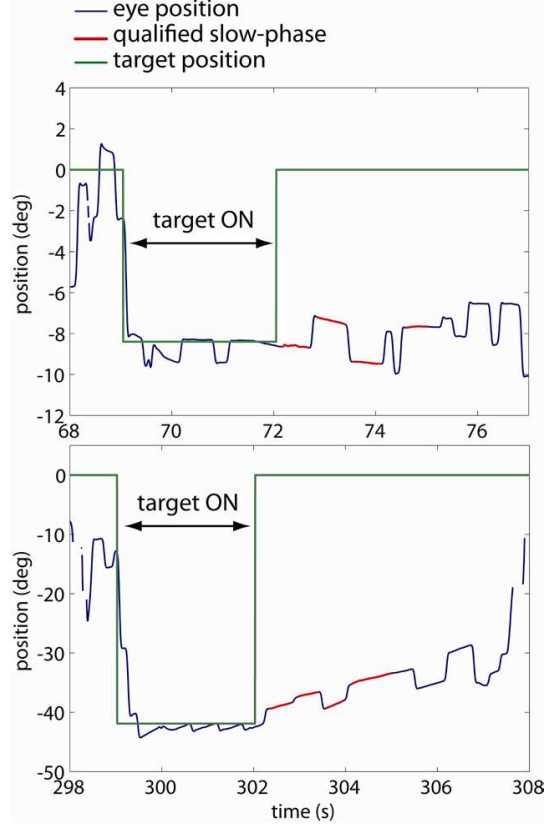

Figure 1-A typical example of eye position traces during fixation periods in the light followed by gaze-holding in darkness. The slow-phases that were selected for later analyses are highlighted in red.

Since the NI is shared by all oculomotor subsystems that generate eye movements to a variety of visual and vestibular stimuli, understanding its function as well as factors that influence it are crucial for studying ocular motor control in general. To characterize the NI, the first step is to measure its time constant. Indeed, a number of studies have addressed this issue by measuring the velocity of the ocular drift towards a null position during gaze holding in darkness. In general subjects were required to maintain fixation on an imaginary target in the dark. The slope of the drift velocity versus position (i.e. the P-V plot) is indicative of the time constant of the integrator. The results of these studies have nevertheless been contradictory. For example Becker and Klein [2] and Hess et al. [3], both using the EOG (electrooculography) technique during gaze holding experiments on healthy human subjects, concluded that when averaged across all subjects, the P-V plot was linear, and the time constant of the NI did not vary with eye position. Whereas Eizenman et al. [4] did the same experiments using video-oculography and showed that the $\mathrm{P}-\mathrm{V}$ curve is nonlinear, at least for eccentric fixations beyond $20^{\circ}$. Magnetic search coil measurements in goldfish during spontaneous scanning of the surroundings also revealed nonlinear P-V plots, suggesting that at least in goldfish integrator function depends on eye position in the orbit [5]. There are also several modeling studies [6-11] that propose the necessity for eye position dependent nonlinearities in the 
NI network, although these predictions have not yet been verified by experimental studies. In this article we present the results of gaze-holding experiments in healthy human subjects, using videooculography technique. We report that in most of the subjects (9 out of 15) the P-V plot was in fact nonlinear, suggesting an eye position dependent integrator function.

\section{METHODS}

Fifteen healthy human volunteers (ages 31.8 \pm 11.2 ) with no known vestibular or ocular motor deficits participated in the experiments and gave written consent after being informed about the experiment. Experiments were approved by the local ethics committee and in accordance with the principles of the Declaration of Helsinki.

Subjects sat upright on a chair, while head movements were minimized by means of a chin rest, and were allowed binocular vision. The chair was surrounded by a con-centric circular drum. The eyes were directed towards a desired position by means of red LED targets installed on the drum. The chin rest was also used to adjust the head height so that the target was aligned vertically at eye level. Eye distance from the drum was $64 \mathrm{~cm}$. Except for the LED targets, the room was completely dark. The LED turned on at a random horizontal position (range $\pm 45^{\circ}$ at $5^{\circ}$ steps), and stayed on for 3s. After this fixation period, the LED turned off and the subject was instructed to maintain fixation at the remembered target for $5 \mathrm{~s}$ after which a beep sound informed the subject to relax. The next target turned on $4 \mathrm{~s}$ after that. The experiment took $\sim 9$ minutes in total and there was a 1 minute rest period in the middle of the session. During this rest period the room light was turned on. None of the subjects reported fatigue after the experiment.

Horizontal and vertical movements of the right eye were monitored at $220 \mathrm{~Hz}$ using a head mounted video camera (EyeSeeCam, Munich, Germany). Only the horizontal eye position is reported here. The center of the pupil was automatically determined by ellipse fits to thresholded images of the eye. Calibration data was collected for each subject during fixation of targets between $\pm 45^{\circ}$ horizontal and $\pm 10^{\circ}$ vertical. Rightward movements and positions are considered positive.

VOG signals were first low-pass filtered at $10 \mathrm{~Hz}(-3 \mathrm{~dB}$ cut-off frequency) with a zero-phase FIR filter and then scaled using calibration data. Note that in all results an eye position of $0^{\circ}$ corresponds to looking straight ahead. Velocity was derived by a custom numerical algorithm that computed the 5-point derivative of the position signal. Fast-phases were identified and discarded using a velocity threshold criteria set at $5 \%$, as per [5]. Selected slow-phases were also visually inspected and any remaining mini-saccades were removed. Only the data during the first 3 s of attempted gaze holding were analyzed to exclude adaptation effects such as nullposition shifts. The first $100 \mathrm{~ms}$ after the off-set of the LED was not analyzed to account for visual feedback delays. Slow-phase segments longer than $700 \mathrm{~ms}$ were split to segments of at least $200 \mathrm{~ms}$ length. Slow-phases shorter than $200 \mathrm{~ms}$ were also not included in analyses. Data points were discarded at the start and end of each slow-phase to allow enough time for filter dynamics to relax, which was enough to also remove the post-saccadic slip and pre-saccadic abnormalities.

Qualifying slow-phases were fit with a straight line based on minimum least squared error criteria (as an example see Fig. 1). The slope of the fitted line defines the drift rate (i.e. velocity) of the given slow-phase, which was then plotted against mean position to obtain $\mathrm{P}-\mathrm{V}$ plots. During gaze holding periods following a saccade, the input-output relationship of a linear neural integrator is given by:
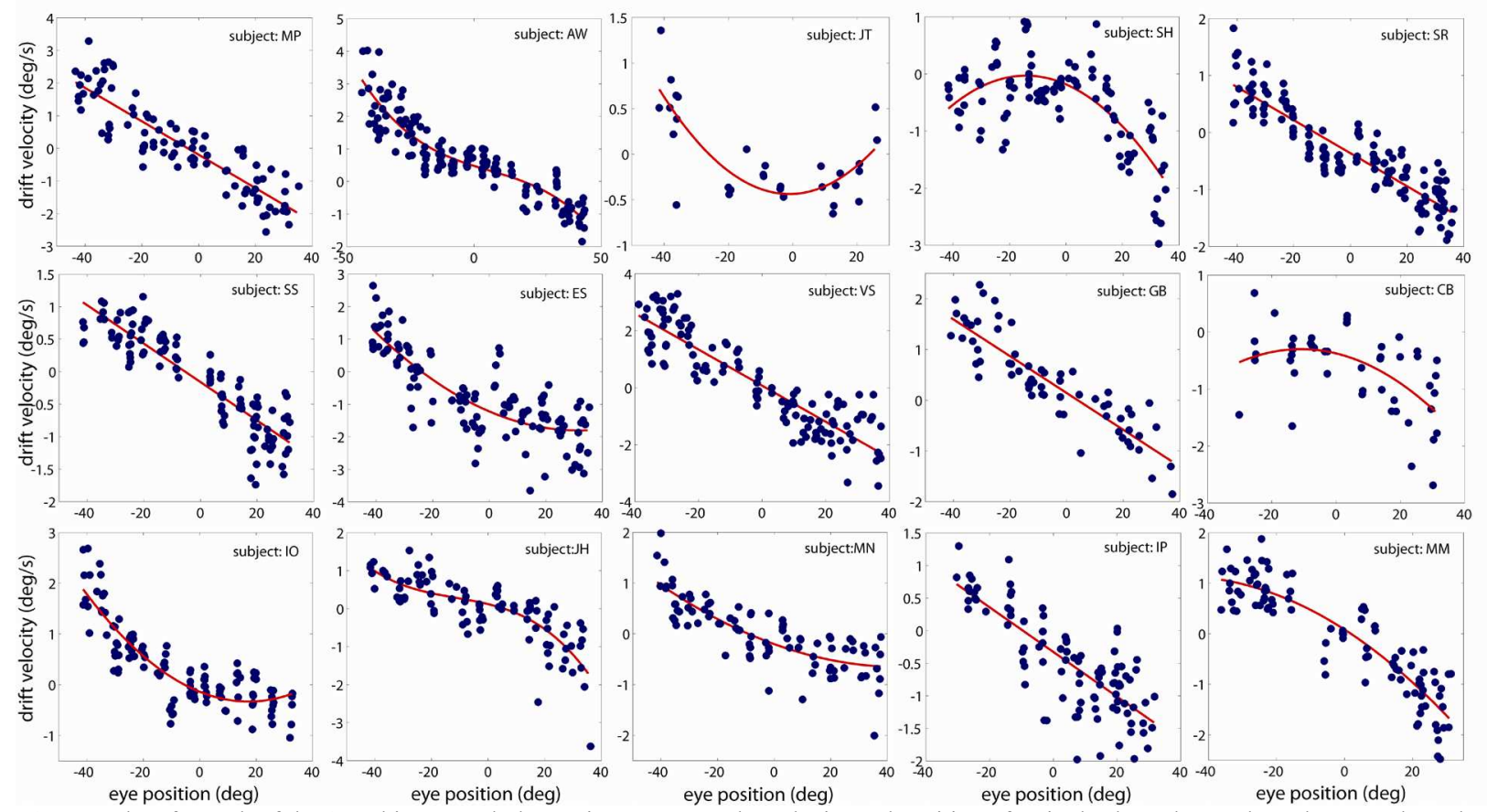

Figure 2- P-V plots for each of the 15 subjects. Each data point represents the velocity and position of a single slow-phase. The red curves show the best fit through the data, based on the criteria mentioned in the Methods. 
TABLE I. SUMMARY OF RESULTS

\begin{tabular}{|l|l|l|l|c|c|}
\hline \multirow{2}{*}{ Subject } & \multicolumn{3}{|c|}{ Variance accounted for by } & \multirow{2}{*}{$\begin{array}{c}\text { Time } \\
\text { each fit }\left(^{2} \text { ) }\right. \\
\text { constant } \\
\text { (s) }\end{array}$} & $\begin{array}{c}\text { Integrator's } \\
\text { null } \\
\text { position (') }\end{array}$ \\
\cline { 2 - 4 } & Linear & Quadratic & Cubic & & -4 \\
\hline MP & $0.79 *$ & 0.79 & 0.79 & 20 & +19 \\
\hline AW & 0.80 & 0.81 & $0.83 *$ & 17 & -26 \\
\hline SH & 0.29 & $0.54 *$ & 0.56 & 36 & -14 \\
\hline SR & 0.30 & $0.52 *$ & 0.53 & 21 & -12 \\
\hline SS & $0.80 *$ & 0.81 & 0.82 & 34 & -5 \\
\hline ES & $0.80 *$ & 0.81 & 0.83 & 34 & -24 \\
\hline VS & 0.62 & $0.67 *$ & 0.68 & 16 & +1 \\
\hline GB & $0.80 *$ & 0.81 & 0.82 & 16 & +4 \\
\hline CB & $0.78 *$ & 0.78 & 0.78 & 28 & - \\
\hline IO & 0.22 & $0.28 *$ & 0.27 & 30 & -5 \\
\hline JH & 0.64 & $0.76 *$ & 0.79 & 20 & +5 \\
\hline MN & 0.57 & 0.60 & $0.62 *$ & 19 & -9 \\
\hline IP & 0.63 & $0.66 *$ & 0.66 & 34 & -9 \\
\hline MM & $0.62 *$ & 0.63 & 0.63 & 30 & +2 \\
\hline
\end{tabular}

Table 1-For each subject the models are compared based on the variance accounted for by the model. Asterisks show the model that was selected based on the criteria explained in the Methods. The time constant and the null position of the integrator are also estimated from the chosen model and given for each subject (see text).

$$
e^{\prime}=k e+v_{0}+v_{\text {sacc }}
$$

which reduces to:

$$
e^{\prime}=k e+v_{0}
$$

for gaze holding in the dark [12]. The integrator time constant can be derived from (2) as $T=-1 / k$ and the null position of the integrator will be $e_{0}=-v_{0} / k$. To analytically characterize the P-V plots, second and third order polynomial models, as well as the linear model in (2) were tried using minimum least squared error criteria. The best model was chosen based on confidence intervals of parameter estimates, the outcome of the F-test (to select from nested models), and the variance accounted for by the model (i.e. the square of adjusted coefficient of correlation, $\mathrm{R}^{2}$ ). Whenever parameter estimates were significantly different from zero $(95 \%$ confidence intervals), and the f-value was greater than $\mathrm{F}\left(0.05,1, \mathrm{~N}_{\text {data points }}\right)$, and there was an improvement in the variance accounted for, the higher order model was selected.

\section{RESULTS}

The P-V plots are displayed in Fig. 2 for all subjects. In some subjects a gaze-evoked nystagmus was observed at more eccentric fixation points (above $35^{\circ}$ ). In the ocular motor range tested $\left( \pm 45^{\circ}\right)$, the $\mathrm{P}-\mathrm{V}$ plots were linear for 6 subjects, and nonlinear for the remaining 9. For subjects with nonlinear drift, the slope of the P-V curve was itself a function of the position, and a time constant is thus defined as the smallest value on the $-1 /$ slope curve. Values are summarized in Table I. The measured time constant values are in agreement with previous human studies $[2,3]$. The higher order terms in nonlinear fits were significant but small, which is because drift rates are rather small over a relatively large ocular range. The nonlinearity that is

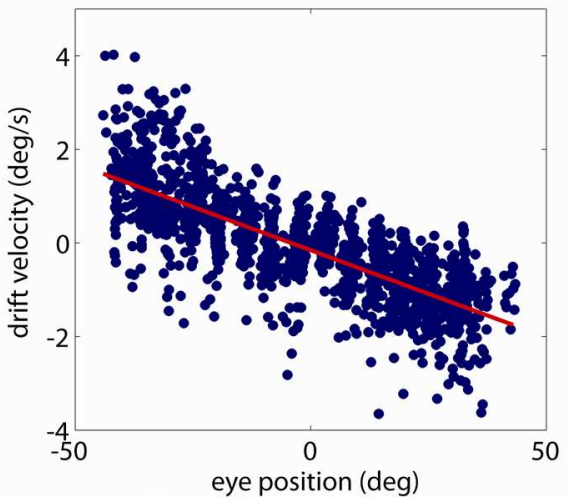

Figure 3- The P-V plot for all subjects, superimposed. Each data point represents the velocity and position of a single slow-phase in a subject. The red curve shows the fit for all subjects. Since there was no uniform nonlinear trend, a cross subject averaging results in a linear model.

observed in subjects is due to higher drift rate at one end of the gaze field and very low or nonexistent drift in the other end. In 2 subjects (AW and $\mathrm{JH}$ ) the rate of change in velocity with position increased at either of the two ends of the gaze field while remaining small in central positions, in agreement with the findings of Eizenman et al. [4]. This means near ideal integration in one part of the gaze field which deteriorates as the eye moves away. Nevertheless in our data we did not see a unified trend in all subjects with nonlinear pattern. A plot of all subjects' data is shown in Fig. 3. Since idiosyncratic nonlinearities did not follow a unified trend, the nonlinear effect cancels out when all the data from all subjects are pooled. Based on the criteria described in the Methods, a linear model is sufficient to describe the cross-subject data, in the range tested. The estimated time constant of this linear integrator is $27 \mathrm{~s}$ and the null position is $4^{\circ}$, i.e. shifted nasally from straight ahead.

\section{DISCUSSION}

Characterization of the neural integrator function in healthy subjects is important for evaluation of patients with ocular-motor symptoms. Such symptoms include gazeevoked, rebound, and congenital nystagmus and are considered to be triggered by impairment of the neural integrator system.

In this study, we tested the linearity of the P-V plots during gaze holding in darkness. Our results show that in the ocular motor range tested $\left( \pm 45^{\circ}\right)$, most subjects have a position-dependent neural integrator. However, the form of this nonlinearity was not the same in all subjects. This could suggest that every subject has a "preferred" gaze region inside which the integrator is trained to function best. Outside of this region the integration time constant decreases, or even approaches instability, as we observed in some subjects (JT, SH, CB). In the same manner, it is possible that subjects who showed a linear trend only have a larger "preferred" range around the straight ahead direction, 
and that integration becomes worse outside of the region that was examined here.

Previous nonlinear identification algorithms, as well as modeling studies by Galiana et al. [7-10] have also proposed that healthy human subjects could exhibit nonlinear integration characteristics during VOR movements. They also suggested that perhaps the neural integrator time constant is highest closer to the origin and declines at lateral positions. This nonlinear behavior could also explain the nonlinear P-V plots during spontaneous nystagmus seen in patients that suffer from acute unilateral vestibular deficits [13]. The integration is accomplished through interconnections of a large network of neurons on either side of the brainstem. In case of a unilateral peripheral lesion, the function of the neural integrator will be affected; its linear

\section{REFERENCES}

[1] A. Skavenski, DA. Robinson, "Role of abducens neurons in vestibuloocular reflex", J Neurophysiol., 36: 724-38, 1973.

[2] W. Becker, HM. Klein, "Accuracy of saccadic eye movements and maintenance of eccentric eye position in the dark", Vision Res., 13: 1021-1034, 1973.

[3] K. Hess, H. Resine, M. Dürsteler, "Normal eye drift and saccadic drift correction in darkness", Neuro-Ophthalmol., 5: 247-252, 1985.

[4] M. Eizenman, P. Cheng, J. Sharpe, R. Frecker, "End-point nystagmus and ocular drift: An experimental and theoretical study", Vision Res., 30: 863-877, 1990

[5] B. Mensh, E. Aksay, D. Lee, H. Seung, D. Tank, "Spontaneous eye movements in goldfish: oculomotor integrator performance, plasticity, and dependence on visual feedback", Vision Res., 44: 711-726, 2004.

[6] L. Optican, D. Zee, "A hypothetical explanation of congenital nystagmus”, Biol. Cybern., 50: 119-134, 1984.

[7] L. Abel, L.F. Dell'Osso, R. Daroff, "Analog model for gaze-evoked nystagmus”, IEEE Trans. Biomed. Eng., BME-25:71-75, 1978.

[8] E. Khojasteh-Lakelayeh, H. Galiana, "A nonlinear model for contextdependent modulation of the binocular VOR", IEEE Trans. Biomed. Eng., 53:986-995, 2006.

[9] E. Khojasteh, H. Galiana, "Implications of gain modulations in brainstem circuits: VOR control system", J. Comput. Neurosci., 27:437-51, 2009

[10] W. Chan, H.L. Galiana, "Modeling the nonlinear context dependency of the neural integrator in the vestibulo-ocular reflex", IEEE Trans. Biomed. Eng., 55:1946-1955, 2008.

[11] E. Khojasteh, H.L. Galiana, "Implications of gain modulation in the binocular VOR circuit: a theoretical study", J. Comput. Neurosci., 27:437-51

[12] M. Goldman, C. Kaneko, G. Major, E. Aksay, D. Tank, H. Seung, "Linear regression of eye velocity on eye position and head velocity suggests a common oculomotor neural integrator", J. Neurophysiol., 88:659-665, 2002.

[13] S. Hegemann, D. Straumann, C. Bockisch, "Alexander's law in patients with acute vestibular tone asymmetry-Evidence for multiple horizontal neural integrators", JARO, 8:551-561, 2007. range could be reduced and its null position shifted, producing more accentuated nonlinear P-V plots.

In summary, on average, a linear representation for the neural integrator can be used in healthy humans, at least over a limited range. Nevertheless, single subjects exhibit nonlinearities that could provide clues to the integrator function in cases with peripheral or central lesions of vestibular and ocular-motor pathways.

Further experiments are needed to investigate the large inter-subject variability that was observed here. As a first step, the intra-subject variability should be studied to determine whether and to what extent results are repeatable for each individual subject. This factor, if true, could explain some of the inconsistencies between the results of different studies. 